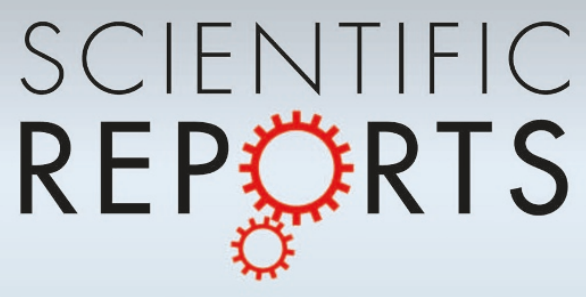

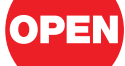

SUBJECT AREAS: PHOSPHORYLATION

APOPTOSIS

CHEMICAL MODIFICATION

NITROSYLATION

Received

7 March 2013

Accepted

23 April 2013

Published

9 May 2013

Correspondence and requests for materials should be addressed to Y.L. (luyan@nju.edu. cn) or P.S. (ppshen@ nju.edu.cn)

* These authors contributed equally to this work.

\section{S-nitrosylation of ERK inhibits ERK phosphorylation and induces apoptosis}

\author{
Xiujing Feng*, Tingzhe Sun*, Yuncheng Bei, Sen Ding, Wei Zheng, Yan Lu \& Pingping Shen
}

State Key Laboratory of Pharmaceutical Biotechnology and Model Animal Research Center (MARC), Nanjing University, Nanjing 210093, China.

Extracellular signal-regulated kinase (ERK) belongs to the mitogen-activated protein kinases (MAPK) superfamily. Aberrant upregulation and activation of ERK cascades may often lead to tumor cell development. However, how ERK is involved in tumor progression is yet to be defined. In current study, we described that ERK undergoes S-nitrosylation by nitric oxide (NO). ERK S-nitrosylation inhibits its phosphorylation and triggers apoptotic program as verified by massive apoptosis in fluorescence staining. The proapoptotic effect of NO induced S-nitrosylation is reversed by NO scavenger Haemoglobin (HB). Furthermore, an S-nitrosylation dead ERK mutant C183A also demolishes the proapoptotic potential of NO and favors cell survival. Therefore, Cys ${ }^{183}$ might be a potential S-nitrosylation site in ERK. In addition, $\mathrm{S}$-nitrosylation is a general phenomenon that regulates ERK activity. These findings identify a novel link between NO-mediated S-nitrosylation and ERK regulation, which provide critical insights into the control of apoptosis and tumor development.

N itric oxide $(\mathrm{NO})$ is a short lived free radical and plays critical roles in the regulation of neuronal, immune, and cardiovascular systems $\mathrm{s}^{1}$. It can be produced in many mammalian cells through a reaction catalyzed by a family of NO synthases (NOS) with many isoforms ${ }^{1,2}$. NO predominantly functions as a messenger or effector molecule and production of NO has been involved in cell death via apoptosis in neurons, macrophages and a variety of tumor cells ${ }^{3}$. The pro-apoptotic effect of $\mathrm{NO}$ is tightly controlled by many cellular events and apoptosis is correlated with increased levels of NO-mediated protein modification ${ }^{4}$. One of the most wellestablished mechanisms of NO-induced modifications is S-nitrosylation ${ }^{5}$. This critical S-nitrosylation can regulate a plethora of biological processes such as cell proliferation, survival and especially apoptosis ${ }^{3,5}$. Although some reports suggested an antiapoptotic role for ERK (extracellular signal-regulated kinases) via S-nitrosylation of caspase-8, caspase-9 and BCL-2 (B-cell lymphoma 2) proteins, many other studies also identified that NO may activate apoptotic processes via distinct mechanisms ${ }^{1,6,7}$. Overproduction of nitric oxide by high levels of exogenous nitric oxide donors often leads to activation of mitochondrial or death receptor mediated apoptotic signaling pathways ${ }^{1,3}$. It has been reported that $\mathrm{NO}$ can impair the mitochondria respiratory chain and induce apoptosis through haeme-nitrosylation of cytochrome $c^{8}$. Treatment of ovarian carcinoma cells with the NO donor nitrosylcobalamin (NO-Cbl) promotes S-nitrosylation of DR4, a member of death receptor family, at the $\mathrm{Cys}^{336}$ residue and consequently activates apoptotic pathways ${ }^{9}$. Furthermore, NO-induced S-nitrosylation and subsequent oxidation of MMP9 (matrix metalloproteinase-9) can lead to enzymatic activation, disruption of extracellular matrix and contribute to one form of apoptosis, termed anoikis ${ }^{10}$. Other reports suggested that in nitrosative cells states NO could probably activate not commonly involved caspases (e.g. caspases- 1 and caspases10) and trigger apoptosis in human colon cancer cells. Nitrosative stress can also initiate apoptosis through activation of mitochondrial pathways, such as release of cytochrome $c$ and endonuclease G, as well as the inhibition of NF- $\mathrm{KB}$ (nuclear factor $\mathrm{\kappa B}$ ) and increased p53 expression ${ }^{11}$.

ERK1/ERK2, also named MAPK3/MAPK1 (mitogen-activated protein kinase) officially, belongs to the mitogen-activated protein kinases superfamily which includes ERK5, JNKs and the p38 MAP kinases ${ }^{12}$. They are activated by tandem phosphorylation of threonine and tyrosine residues on the dual-specificity motif (T-E-Y) and involved in the regulation of cell cycle progression, proliferation, cytokinesis, transcription, differentiation, senescence and apoptosis ${ }^{13}$. Many studies show that ERK1/2 pathway possesses anti-apoptotic functions, depending on the cell type and stimuli. The mechanism of ERK1/2 mediated cell survival is primarily through increased activity of anti-apoptotic proteins such as Bcl-2, Mcl-1, IAP (inhibitor of apoptosis), and repressed pro-apoptotic proteins such as Bad and Bim ${ }^{14}$. ERK1/2 activation is regulated by various mechanisms, including downstream scaffolds, localization and inhibitors of ERK/MAPK signaling ${ }^{12,15}$. However, the exact relation between Snitrosylation and ERK1/ERK2 pathway has yet to be uncovered. 
A

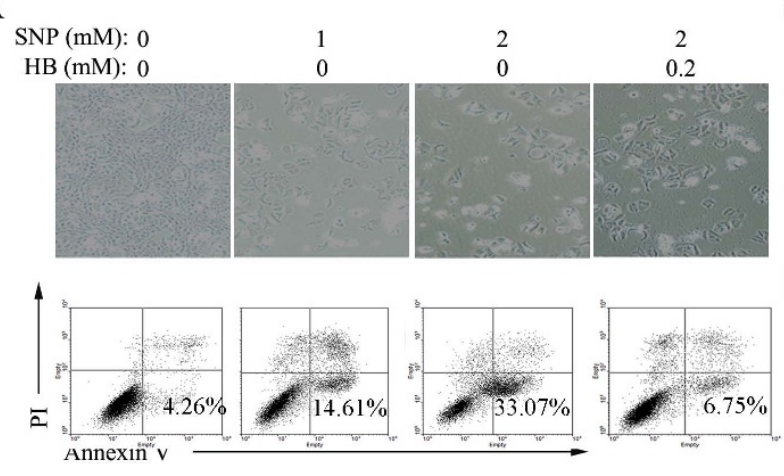

B

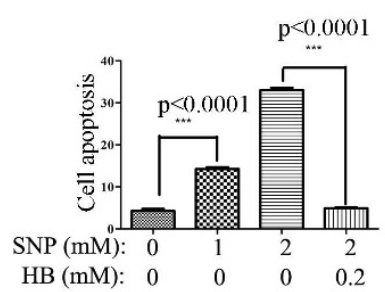

$\mathrm{D}$

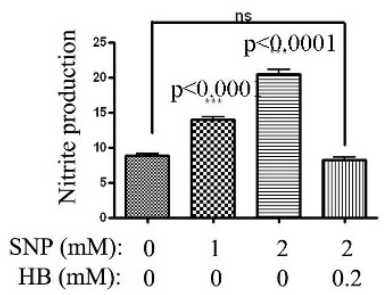

$\mathrm{C}$

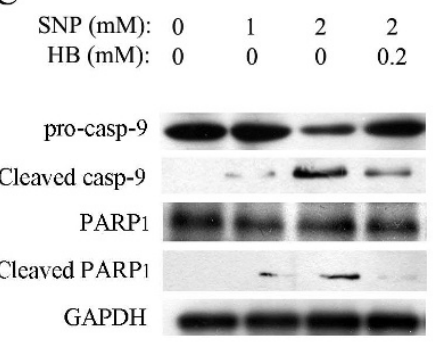

$\mathrm{E}$

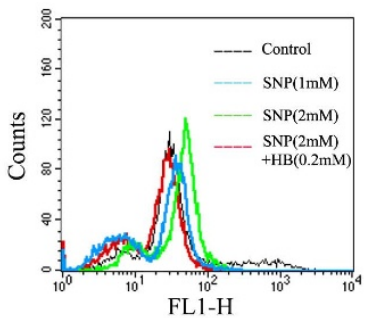

Figure 1 | Elevated NO levels induce apoptosis in MCF-7 cells. (A-B) SNP induces significantly elevated apoptosis and HB reverses the effect. (C) SNP accelerates the process of procaspase-9 and PARP-1. (D-E). Quantification of cell outer NO levels using Griess method and intracellular NO levels using DAF fluorescence staining.

In current study, we aim to investigate the role of S-nitrosylation of ERK1/2 in the regulation of phosphorylation of ERK1/2 in nitric oxide-induced apoptosis of MCF-7 cells. Abnormal elevation of pERK has been described in numerous tumor cells. We found that nitric oxide decreases p-ERK level in NO-induced MCF-7 cell apoptosis. The mechanism by which nitric oxide mediates its regulation of p-ERK involves S-nitrosylation of the protein. Mutational analysis showed that the Cys ${ }^{183}$ is vital for S-nitrosylation of ERK1/2 and NOinduced MCF-7 cell apoptosis. These findings uncover a new mechanism of nitric oxide-mediated regulation of ERK1/2 that could be important in apoptosis resistance and the development of tumor cells.

\section{Results}

Apoptosis and caspase activation induced by NO donor SNP. To study the role of NO in the context of apoptosis, we investigated the apoptotic responses in MCF-7 breast cancer cells. Cells were treated with different concentrations of NO donor SNP ranging from 0$2 \mathrm{mM}$ either in the presence or absence of NO scavenger heamoglobin (HB). We found a dose dependent increase in the apoptotic fraction of MCF-7 cells at $12 \mathrm{~h}$ after NO treatment as indicated by elevated fluorescence in Annexin-V/PI staining (figs. $1 \mathrm{~A}$ and B). Significant apoptotic responses could be observed under the treatment with $1 \mathrm{mM} \mathrm{SNP}$ and the apoptotic fraction was further amplified with $2 \mathrm{mM} \mathrm{SNP} \mathrm{(figs.} 1 \mathrm{~A}$ and B). The procaspase-9 was proteolytically processed and cleaved PARP-1 (Poly [ADP-ribose] polymerase 1) was also detected indicating an apoptotic process (fig. 1C). We monitored cellular NO levels using Griess method. SNP can break down to release NO and shows a dose-dependent increase (fig. 1D). However, this effect can be reversed by HB showing that NO can be cleaned effectively (fig. 1). We also monitored the intracellular NO level by DAF fluorescence staining and found that the NO level also increases (fig. 1E). However, the apoptotic responses were significantly abrogated when cellular levels of $\mathrm{NO}$ were cleared with $\mathrm{HB}$ implying that elevated $\mathrm{NO}$ might trigger a massive apoptosis in MCF-7 cells (figs. 1 A-D). These results suggest that NO play a proapoptotic role in MCF-7 cells.
NO inhibits the phosphorylation of ERK. There is considerable evidence that ERK MAPK pathway promotes cell survival and much efforts have been focused on this pathway ${ }^{12,15}$. ERK is dually phosphorylated at a conserved TEY motif ${ }^{16}$. Therefore, we examined the effect of NO on the phosphorylation status of ERK. Fig. 2A showed that although a slight increase in phosphorylation of ERK is evident at early time points, the phosphorylation level of ERK was significantly diminished at $4 \mathrm{~h}$ and $5 \mathrm{~h}$ after SNP treatment. A famous ERK substrate ELK1 was also phosphorylated at early time points but decreased later following similar dynamics with levels of phosphorylated ERK (fig. 2A). The inhibitory effect was further augmented in a dose dependent manner as confirmed by immunological blot (fig. 2B). It has been shown that phosphorylated and activated ERK (p-ERK) is able to phosphorylate PPAR $\gamma$ on Ser112. To further investigate the inhibitory effect of NO on ERK phosphorylation and kinase activity, we transfected MCF-7 cells using polycationic liposome transfection reagent Lipofectamine ${ }^{\mathrm{TM}} 2000$ with either wild type (WT) ERK or a constitutive active form (TEYEED) harboring a point mutation. The transfected cells with wild type ERK showed basal PPAR $\gamma$ phosphorylation while those with the active mutants (TEY-EED) presented a much higher phosphorylation level for PPAR $\gamma$ (fig. 2C). However, NO donor SNP failed to decrease the kinase activity of the mutational form TEY-EED (fig. 2C). Furthermore upon SNP treatment, cells transfected with wild type ERK caused substantially increased apoptosis (from 9.38\% to $56.94 \%$ ) while transfectants with kinase active mutants (TEYEED) partially reverse the apoptotic effect (fig. 2D, from $56.94 \%$ to $20.47 \%$ ). We also verified the SNP mediated inhibition of ERK phosphorylation in HeLa cells (fig. S1). We further found that adding NO donor SNP can trigger cellular apoptosis implying that this inhibitory effect is not cell type specific (fig. S1). These results indicated that SNP was able to inhibit ERK phosphorylation and promote apoptosis.

NO can induce S-nitrosylation of ERK. S-nitrosylation is a relatively abundant process in biological milieu on exposure to NO donor (e.g. SNP). To determine whether NO can nitrosylate ERK, detergent solubilized extracts were analyzed by biotin switch assay. 
A

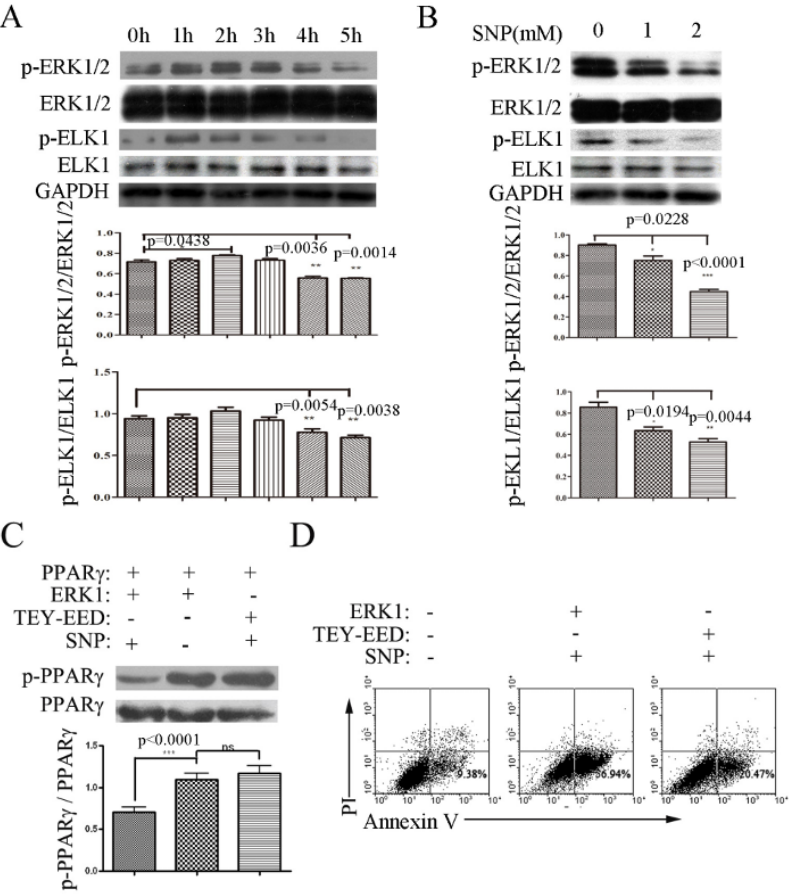

Figure $2 \mid$ NO inhibits the phosphorylation of ERK and promotes MCF-7 cells apoptosis. (A) Cells were treated with $1 \mathrm{mM} \mathrm{SNP}$ and the phosphorylation of ERK was examined by immunoblots. (B) ERK phosphorylation was quantified in cells treated with SNP (0 to $2 \mathrm{mM})$. (C) Wild type and constitutively active mutant of ERK (TEY-EED) were used to investigate the inhibitory role of NO on ERK phosphorylation.

(D) Apoptotic effects were examined using flow cytometry.

Extracts from 293T cells were treated with Cys-NO in the presence or absence of ascorbate for $30 \mathrm{~min}$ and then subject to immunoblots. Snitrosylated cysteines are recognized by ascorbate-dependent SNO cleavage followed by biotinylation of free thiols. We found that ERKSNO (i.e. S-nitrosylated ERK) could be detected when ascorbate was added (fig. 3A). In addition, S-nitrosylation was further amplified with increasing doses of SNP (from 0 to $2 \mathrm{mM}$, fig. 3B). These results suggested that NO was able to S-nitrosylate ERK in a dose dependent manner.

Mutations at Cys ${ }^{183}$ prevents ERK S-nitrosylation. There exist six cysteine residues which allow for potential S-nitrosylation of ERK. A preliminary computational prediction indicated that cysteine 183 is the most possible S-nitrosylation site (fig. S2). To further ascertain which cysteine residue is S-nitrosylated experimentally, we constructed all six transfected ERK mutants in MCF-7 cells harboring one cysteine replaced by alanine (i.e. $\mathrm{C}$ to A mutation, fig. 4A). The expression of endogenous mutants were verified by immunoblots using anti-HA antibody. The results in fig. 4A showed that ERK
A

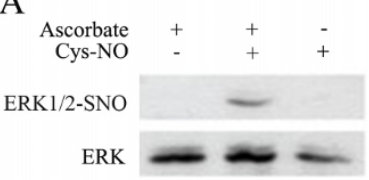

B

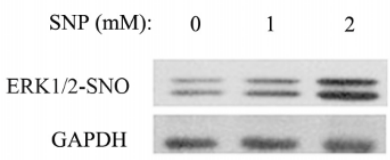

Figure 3 NO induces S-nitrosylation of ERK. (A) Extracts from 293T cells treated with Cys-NO in the presence or absence of ascorbate and the level of ERK-SNO was detected by S-nitrosylation (Immunoprecipitation) assay in vitro. (B) $293 \mathrm{~T}$ cells were treated with SNP (0 to $2 \mathrm{mM}$ ) and the level of ERK-SNO was detected by S-Nitrosylation Assay (Biotin-Switch). was detectable in all cells with distinctive ERK mutants. We further investigated the inhibition of S-nitrosylation of ERK between C183A and C271A and found that S-nitrosylated ERK is severely attenuated in C183A transfected mutants (fig. 4B) and this is consistent with the software prediction that $\mathrm{C} 183 \mathrm{~A}$ site has a better score (fig. s2). We then transfected 293T cells with C183A mutant and BST assay showed a significantly abolished S-nitrosylation of ERK (fig. 4C). These results clearly indicate that the 183 cysteine residue might be a principle S-nitrosylation site for ERK.

S-nitrosylation of ERK inhibits ERK phosphorylation and promotes apoptosis. To establish the relation between ERK Snitrosylation and phosphorylation, we further transfected MCF-7 cells with wild type ERK or C183A mutant (i.e. S-nitrosylation dead form) either in the presence or absence of SNP using Lipofectamine ${ }^{\mathrm{TM}} 2000$. The endogenously transfected ERK was verified by immunoblots (fig. 5A). The results showed that the phosphorylation of ERK was reduced when SNP was added (fig. 5A). However, SNP failed to inhibit the phosphorylation of the C183A mutant implying that blocking ERK S-nitrosylation might favor ERK phosphorylation (fig. 5A). The kinase activity assay showed that ERK kinase activity reduced under SNP stress. However, SNP failed to inhibit the ERK kinase activity of the C183A mutant. This showed that ERK S-nitrosylation directly affected the ERK phosphorylation (fig. 5B). PPAR $\gamma$, a phosphorylation substrate of p-ERK was less phosphorylated following SNP treatment while the phosphorylation status in transfectants with the C183A mutant form of $\mathrm{p}$ ERK was not affected (fig. 5C). Meanwhile, the proapoptotic effect of SNP treatment was also reversed by transfection with C183A mutant as evident by less procaspase- 9 processing (fig. 5D). These effects were also confirmed by fluorescence staining where cells with C183A mutant was unable to commit apoptosis under SNP treatment compared with transfectants with wild type ERK (figs. 5E). There results indicate that S-nitrosylation of ERK inhibits ERK phosphorylation and favors apoptosis.

S-nitrosylation of ERK under other stressed conditions. To identify whether S-nitrosylation of ERK is a universal effect under stressed conditions, we applied other stress-inducing agents including hydrogen peroxide and TNF- $\alpha$ and performed immunoblotting experiments. The results showed that ERK was also S-nitrosylated under both stressed conditions (fig. 6). Meanwhile, the phosphorylation of C183A mutant cannot be inhibited under TNF- $\alpha$ and $\mathrm{H}_{2} \mathrm{O}_{2}$ treatment indicating that $\mathrm{S}$-nitrosylation of ERK is probably interrupted (fig. S3). In addition, the NO necessary for apoptotic induction is generated intrinsically as evidenced by elevated $\mathrm{NO}$ levels under $\mathrm{H}_{2} \mathrm{O}_{2}$ treatment (fig. S4). TNF- $\alpha$ treatment can also

A

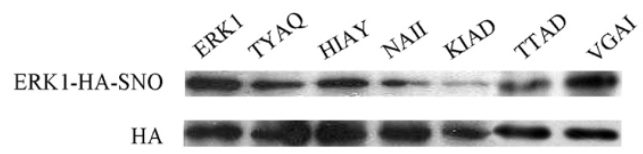

B

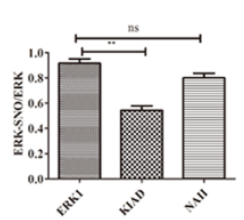

C

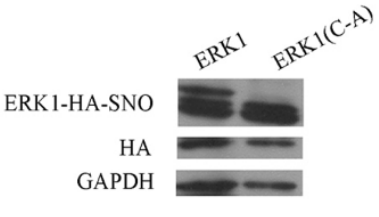

Figure $4 \mid$ Point mutations on cysteine residues inhibit S-nitrosylation. (A) For mutants (TYAQ: C82A; HIAY: C144A; NAII: C271A; KIAD: C183A; VGAD: C233A and TTAD: C178A) were explored for potential inhibition of S-nitrosylation. (B) The level of ERK-SNO was normalized by total ERK1/2. (C) KIAD mutant was examined for S-nitrosylation. 
A

$\begin{array}{rrrrr}\text { ERK1-HA(C-A) } & - & - & - & + \\ \text { ERK1-HA } & - & + & + & - \\ \text { SNP } & - & - & + & +\end{array}$

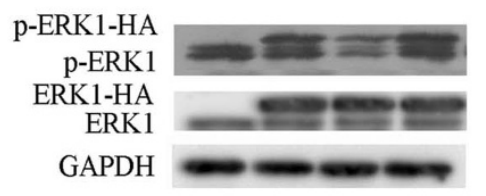

$\mathrm{D}$

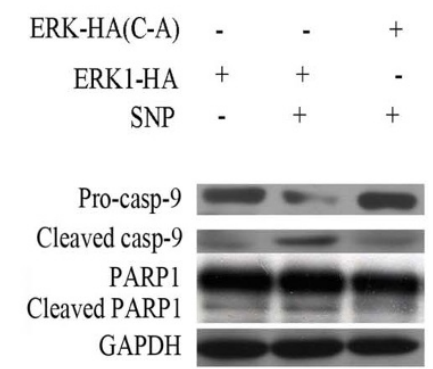

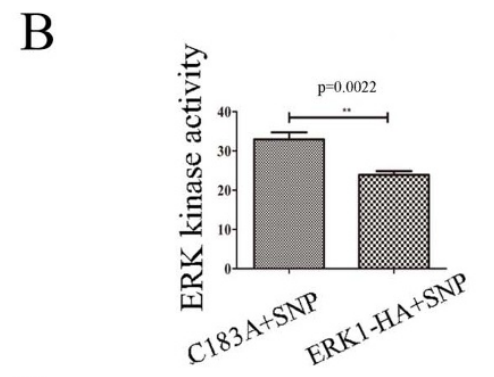

$\mathrm{C}$

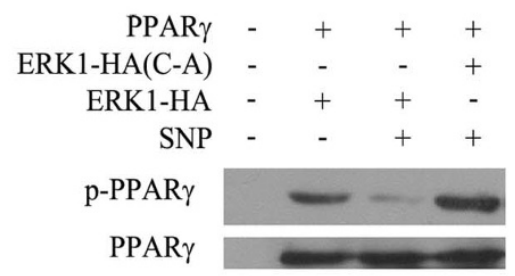

$\mathrm{E}$
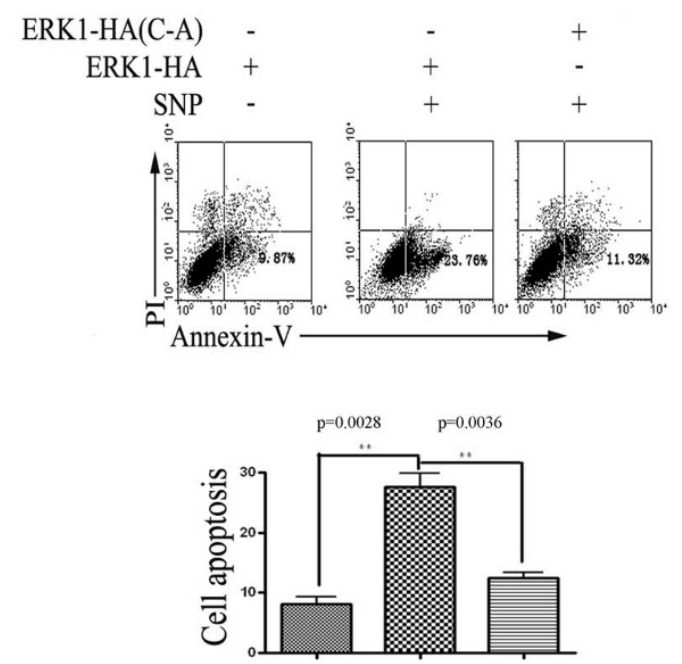

Figure 5 | S-nitrosylation of ERK1 directly inhibits ERK phosphorylation and promotes apoptosis. (A) MCF-7 cells were transfected with ERK1-HA or ERK1 mutant form (C183A), the phosphorylation of ERK was determined by immunoblots. (B) ERK kinase activity was detected using the ERK kinase assay kit. $\mathrm{P}=0.0022(\mathrm{C}) \mathrm{PPAR} \gamma$, a phosphorylation target of ERK was examined for phosphorylation status. (D) MCF-7 cells were transfected with either wild type or C183A mutant ERK and the process of procaspase- 9 was quantified by immunoblots. (E) Apoptotic effects of SNP in MCF-7 cells transfected with either wild type or C183A mutant ERK were determined by flow cytometry.

impair ERK and $\operatorname{PPAR} \gamma$ phosphorylation, promote caspase-9 proteolytic degradation and induce apoptosis whereas transfection with the S-nitrosylation dead C183A mutant can attenuate these proapoptotic effects (fig. S5). These results suggested that Snitrosylation of ERK by intrinsic NO production is a general effect that dictates apoptotic program under stressed conditions.

\section{Discussion}

Considerable efforts have been spent on targeting MEK-ERK-MAPK pathway owing to its potential in promoting cell survival, proliferation and metastasis ${ }^{12}$. S-nitrosylation is a universal phenomenon under nitrosative stress, however, the exact role of S-nitrosylation

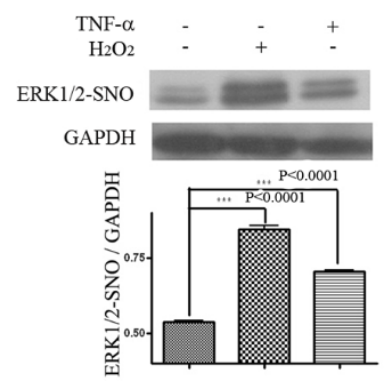

Figure 6 | S-nitrosylation of ERK under other stressed conditions. MCF-7 cells were treated with $0.5 \mathrm{mM} \mathrm{H}_{2} \mathrm{O}_{2}$ or $50 \mathrm{ng} / \mathrm{mL}$ TNF- $\alpha$, respectively for 6 hours and S-nitrosylation status was determined by immunoblots. in ERK regulation has never been identified. Our current study describes that S-nitrosylation of ERK contributes to the cellular commitment to apoptosis by inhibiting ERK phosphorylation and therefore adds another complexity to apoptotic regulation. We showed that under nitrosative stress, NO donor SNP can initiate apoptotic program in a dose dependent manner (fig. 1). As ERK phosphorylation favors cell survival, we further evaluated the phosphorylation and activation status of ERK and found that ERK phosphorylation was abrogated following SNP treatment (fig. 2). We speculated that under nitrosative stress, ERK might be S-nitrosylated and this effect was verified by biotin switch assay (fig. 3) and then we identified Cys ${ }^{183}$ might be a potential nitrosylation site (fig. 4). For the relationship between S-nitrosylation and phosphorylation of ERK, we showed that S-nitrosylation was directly sabotage ERK phosphorylation and inhibit ERK kinase activity and therefore triggers apoptotic program (fig. 5). However, the S-nitrosylation may not be restricted to SNP treatment, while oxidative and pro-inflammatory stress can also induce ERK S-nitrosylation through induction of intrinsic NO (fig. 6). These results substantiate the critical role of S-nitrosylation in ERK regulation.

S-nitrosylation is a ubiquitous posttranslational modification with diverse biological outcomes and the NO displays both stimulatory or inhibitory effects in the context of apoptosis ${ }^{1,3,4}$. In hepatocytes, Snitrosylation of caspase- 8 blocked Bid activation and TNF- $\alpha$ induced apoptosis $^{1,6,7,17}$. NO donors were also found to inhibit assembly of apoptosome $e^{1,6,7,17}$. Other reports showed that S-nitrosylation of cytochorme $c$ and GAPDH facilitates apoptosome formation and initiates a p53 proapoptotic pathway respectively ${ }^{8,18}$. The opposing tasks 
by NO is probably ascribed to the availability of enzymes, timing of apoptotic stimuli, redox state, donor doses, spatial location of key reactants and interactions with other molecules ${ }^{19}$. Therefore, a combination of complex factors may determine the net effect of NO in a specific cell type ${ }^{19}$. Noticeably in an investigation of dose effect, Maejima et al. found that low doses of NO donor SNAP favor cell survival while high doses may reduce cell viability ${ }^{20}$. Another report also showed that adenovirus mediated transfer of inducible NOS (iNOS) also contribute to elimination of various tumor cells ${ }^{21-23}$. The dual effects of NO display concentration dependence. Low levels of NO produced by tumor microenvironment favor tumor cell survival while tumor cells with high NO levels are dedicated to cell death $^{4}$. Given a proapoptotic role of NO, many possibilities exist by triggering tumor cells to produce NO. Besides transfection, IFN- $\gamma$ has also been linked to iNOS expression and presented in clinical therapy of ovarian carcinoma ${ }^{24}$. Therefore, NO mediated regulation may function as a promising adjuvant in cancer therapy. Our current study evaluated the critical role of S-nitrosylation of ERK in apoptotic ${ }^{1}$ induction and shed light on physiological and pharmacological manipulation of apoptotic processes in tumor cells.

Mitogen-activated protein kinase (MAPK) cascades, in particular the ERK cascades, are principle signaling pathways that dictate homeostatic cell proliferation and survival ${ }^{12,25}$. Abnormal elevation in ERK activities contribute to numerous diseases and tumor cell metastasis ${ }^{12}$. We argue that ERK S-nitrosylation provides an intrinsic way to eliminate tumor cells via cell death pathways. Noticeably, the MCF-7 cell lines harbor numerous defects in apoptotic execution $^{26-30}$. In current study, SNP treatment unleashes the apoptotic potential of death resistant cells and therefore articulates a critical modification in cell death regulation and probably tumor cell intervention. We note that S-nitrosylation of ERK impedes dual ERK phosphorylation on conserved threonine 202 and tyrosine 204 sites known as TEY motif (fig. 5). A potential S-nitrosylation site of ERK was identified at $\mathrm{Cys}^{183}$ (fig. 4). Owing to proximity in location, Snitrosylation at $\mathrm{Cys}^{183}$ probably present a spatial barrier to dual phosphorylation of ERK, resulting in functional silencing of ERK. Noticeably, there is overwhelming frequency in which ERK pathways are aberrantly activated in cancer cells and much efforts have been spent on targeting and inhibiting this pathway. Previous approaches primarily cover the inhibitors of upstream ERK activators such as Raf/Ras and $\mathrm{MEK}^{12}$. However, in our current study, we described how S-nitrosylation can exert tight controls over ERK itself. Therefore, we suggest that modulation of S-nitrosylation or cellular redox state can indirectly dictate the functional status of ERK and in turn, regulate various cellular processes such as proliferation, inflammation, cell survival and especially tumor cell metastasis.

The majority of ERK substrates are transcription factors ${ }^{12,31}$. Activated ERK can translocate to nuclear and regulate a myriad of proteins. For example, Ets family transcription factors are phosphorylated by ERK leading to altered gene expression ${ }^{31}$. Therefore, inhibition of ERK nuclear translocation might be to some extent a potential way to protect normal cells from tumor transformation. To current knowledge, ERK has no well-defined nuclear localization sequence (NLS) ${ }^{32}$. However, following activation, some phosphorylation sites promote ERK translocation and nuclear import ${ }^{33}$. We found that ERK S-nitrosylation inhibits its phosphorylation. Therefore, ERK S-nitrosylation may potentially reduce ERK nuclear translocation (data not shown) and ultimately providing another promising way to the targeted intervention of ERK pathways.

In summary, our data provide evidence that NO serves as a positive regulator in apoptotic program. NO exerts this effect through its capacity of S-nitrosylating ERK and inhibiting ERK phosphorylation. The S-nitrosylation of ERK might be a general phenomenon under many stressed conditions and aid in normal cell protection. Since ERK is abnormally upregulated in numerous diseases and tumor cells, targeting ERK might via NO mediated S-nitrosylation be one of the key regulations to terminate cancer cell survival and proliferation. Our finding on ERK regulation by NO mediated Snitrosylation may possess important implications in prevention of carcinogenesis.

\section{Methods}

Reagents and antibodies. DMEM were supplied by Gibco (Grand Island, N.Y.), Site-directed Mutagenesis kit (SBS, beijing, China), TNF- $\alpha$ (Peprotech), the MMTS (methyl-methyl-thiomethyl sulfoxide) and streptavidin-agarose (Fluka); biotinHPDP(Pierce); Lipofectamine ${ }^{\mathrm{TM}} 2000$ (Invitrogen). 3-Amino,4-aminomethyl-2', $7^{\prime}$ difluorescein, diacetate (DAF-FM-DA), NO donor SNP, NO scavenger HB, hydrogen peroxide (Haimen, Jiangsu, China). All chemicals were purchased from Sigma (St. Louis, MO) unless otherwise specified. ERK1/2 (L352) pAb (bs1112), p-ERK1/2 (T202/Y204) pAb (bs5016), ELK1(H377) pAb (bs1105) and p-ELK1(S383) pAb (Bioworld Biotechnology); HA antibody (Haimen, Jiangsu, China); anti-caspase-9 (\#9502), cleaved caspase-9 (ASP330) (\#9501), PARP1 (Cell Signaling technology); PPAR $\gamma$ (E-8) (sc-7273), protein A-agarose (Santa Cruz Biotechnology); phosphoPPAR $\gamma$ (Millipore); S-nitrosocysteine (Sigma); GAPDH (KangChen, Shanghai, China ). ERK kinase assay kit (GMS50056, USA).

Cell culture. MCF-7 cells (Shanghai Institutes for Biological Sciences, Chinese Academy of Sciences, Shanghai, China), HER 293T cells were cultured in Dulbecco's modified Eagle's medium (Wisent), supplemented with 10\% FBS (Wisent), 2 mM Lglutamine, 100 units $/ \mathrm{ml}$ Penicillin and $100 \mathrm{unit} / \mathrm{ml}$ Streptomycin in $5 \% \mathrm{CO}_{2}$ at $37^{\circ} \mathrm{C}$

Site-directed mutagenesis of ERK1 and p-ERK1 constructed. All the mutant ERK1 plasmids were constructed using the QuikChange XL site-directed mutagenesis kit and the mutant plasmids were confirmed by automated nucleotide sequencing. The primers used in the paper were described in Supplementary Table 1.

Plasmid and transfection. The human ERK1 tagged with HA plasmid was generously provided by Philippe Lenormand (Centre de iochimie-CNRS UMR 6543, Universite de Nice). ERK1 was in a retroviral vector, PLPCX. The authenticity of all constructs was verified by DNA sequencing. Transient transfection was performed using Lipofectamine ${ }^{\mathrm{TM}} 2000$ reagent according to the manufacturer's instructions. The amount of DNA was normalized in all transfection experiments with PLPCX. Expression of proteins was verified by Western blotting or immuno-precipitation.

Apoptosis assay. After stimulated for 12 hours, cells were harvested and washed twice with cold PBS, and then were treated with fluorescein isothiocyanate (FITC)conjugated Annexin V and a Prodium Iodide (PI) kit (Immunotech, Marcelle, France) for $30 \mathrm{~min}$ in dark. The cells were analyzed using flow cytometry and a total of 10,000 cells per sample were analyzed in a diparametric plot (FL1 for log FITC and FL3 for $\log$ PI) to determine the percentage of phosphatidylserine (PS)-externalized AnV + PI- (high FITC/low PI) apoptotic cells and PI + (low FITC/high PI-plus-high FITC/high PI) necrotic cells.

NO detection. Intracellular NO production was determined by flow cytometry and fluorescence microscopy using NO-specific probe 3-Amino,4-aminomethyl-2' $7^{\prime}$ difluorescein,diacetate (DAF-FM-DA). For further flow cytometric analysis, cells were treated with SNP or HB for 12 hours, then cells incubated with the probe for $30 \mathrm{~min}$ at $37^{\circ} \mathrm{C}$, after which they were washed, resuspended in PBS, and analyzed for DAF fluorescence. Also, NO production was confirmed by measuring its nitrite byproduct using Griess assay. Cell supernatants $(50 \mu \mathrm{L})$ were mixed with equal amount of Griess reagent for $15 \mathrm{~min}$ at room temperature. The optical density of the samples was measured a spectrophotometer with absorbance set at $540 \mathrm{~nm}$. Sodium nitrite was used as a standard.

S-nitrosylationassay (Biotin-Switch). S-nitrosylated ERK1/2 was detected using the Biotin-Switch assay introduced by Jaffrey with a few improvements. Cells were lysed in lysis buffer (25 mM Hepes, $50 \mathrm{mM} \mathrm{NaCl}, 0.1 \mathrm{mM}$ EDTA, 1\% NP-40, $0.5 \mathrm{mM}$ Phenylmethylsulfonyl fluoride [PMSF] and $0.1 \mathrm{mM}$ neocuproine [ $\mathrm{pH}$ 7.4]). The protein amounts were determined using the BCA protein assay (Pierce). $1.2 \mathrm{mg}$ Protein were mixed with $1.8 \mathrm{~mL}$ blocking buffer (2.5\% SDS, $0.1 \%$ MMTS in HEN buffer) at $50^{\circ} \mathrm{C}$ for 1 hour to S-methylthiolate each cysteine thiol with Smethylmethane thiosulfonate (MMTS). After removing excess MMTS by acetone precipitation, samples were then reduced to thiols and biotinylated by $30 \mu \mathrm{L}$ reducing buffer ( $1 \%$ SDS, $30 \mathrm{mM}$ from sinapic, $4 \mathrm{mM}$ Biotin-HPDP from Pierce). Then the biotinylated proteins were pulled down by streptavidin-agarose beads, eluted by SDS sample buffer and subjected to western blot analysis.

S-nitrosylation (immuno-precipitation) assay. Cells were lysed in lysis buffer and $300 \mu \mathrm{g}$ proteins were incubated with $0.3 \mu \mathrm{g}$ anti-S-Nitro-Cysteine (SNO-Cys) in dark at $4{ }^{\circ} \mathrm{C}$ for 2 hours. Then $5 \mu \mathrm{L}$ protein A/G Plus-agarose beads and $500 \mu \mathrm{L}$ PBS were added into overnight at $4{ }^{\circ} \mathrm{C}$ with gentle rotation. Immune complex were then separated by SDS/PAGE and subjected to Western blot analysis.

ERK kinase activity assay in vitro. The wild type ERK1-HA and mutant type C183A plasmid were transfected into MCF-7 cells and after 24 hours the cells were incubated with $1 \mathrm{mM} \mathrm{SNP}$ for 4 hours. Then ERK kinase activity was detected using an ERK1/2 
kinase assay kit according to the manufacture's instructions (GENMED SCIENTIFICS INC.U.S.A)

Western blotting. Equal amount of protein fractions or agarose beads were mixed with SDS sample buffer, separated on $10 \%$ SDS-polyacrylamid gels, and transferred to nitrocellulose membranes. Then, the membrane sheets were incubated with antibody and visualized by standard chemiluminescence.

1. Circu, M. L. \& Aw, T. Y. Reactive oxygen species, cellular redox systems, and apoptosis. Free radical biology \& medicine 48, 749-762 (2010).

2. Muntane, J. \& la Mata, M. D. Nitric oxide and cancer. World journal of hepatology 2, 337-344 (2010)

3. Wang, Y., Chen, C., Loake, G. J. \& Chu, C. Nitric oxide: promoter or suppressor of programmed cell death? Protein \& cell 1, 133-142 (2010).

4. Lechner, M., Lirk, P. \& Rieder, J. Inducible nitric oxide synthase (iNOS) in tumor biology: the two sides of the same coin. Seminars in cancer biology 15, 277-289 (2005).

5. Lillig, C. H. \& Holmgren, A. Thioredoxin and related molecules--from biology to health and disease. Antioxidants \& redox signaling 9, 25-47 (2007).

6. Kim, Y. M. et al. Nitric oxide prevents tumor necrosis factor alpha-induced rat hepatocyte apoptosis by the interruption of mitochondrial apoptotic signaling through S-nitrosylation of caspase-8. Hepatology 32, 770-778 (2000).

7. Zech, B., Kohl, R., von Knethen, A. \& Brune, B. Nitric oxide donors inhibit formation of the Apaf-1/caspase- 9 apoptosome and activation of caspases. The Biochemical journal 371 (2003).

8. Schonhoff, C. M., Gaston, B. \& Mannick, J. B. Nitrosylation of cytochrome c during apoptosis. The Journal of biological chemistry 278, 18265-18270 (2003)

9. Tang, Z., Bauer, J. A., Morrison, B. \& Lindner, D. J. Nitrosylcobalamin promotes cell death via S nitrosylation of Apo2L/TRAIL receptor DR4. Mol Cell Biol 26, 5588-5594 (2006)

10. Gu, Z. et al. S-nitrosylation of matrix metalloproteinases: signaling pathway to neuronal cell death. Science 297, 1186-1190 (2002).

11. Kroncke, K. D. Nitrosative stress and transcription. Biological chemistry 384, 1365-1377 (2003).

12. Roberts, P. J. \& Der, C. J. Targeting the Raf-MEK-ERK mitogen-activated protein kinase cascade for the treatment of cancer. Oncogene 26, 3291-3310 (2007).

13. Dong, C., Waters, S. B., Holt, K. H. \& Pessin, J. E. SOS phosphorylation and disassociation of the Grb2-SOS complex by the ERK and JNK signaling pathways. The Journal of biological chemistry 271, 6328-6332 (1996).

14. Youle, R. J. \& Strasser, A. The BCL-2 protein family: opposing activities that mediate cell death. Nature reviews. Molecular cell biology 9, 47-59 (2008).

15. Almog, T. \& Naor, Z. Mitogen activated protein kinases (MAPKs) as regulators of spermatogenesis and spermatozoa functions. Molecular and cellular endocrinology 282, 39-44 (2008)

16. Davis, R. J. MAPKs: new JNK expands the group. Trends in biochemical sciences 19, 470-473 (1994).

17. Tzeng, E. et al. Adenoviral transfer of the inducible nitric oxide synthase gene blocks endothelial cell apoptosis. Surgery 122, 255-263 (1997).

18. Sen, N. et al. Nitric oxide-induced nuclear GAPDH activates p300/CBP and mediates apoptosis. Nature cell biology 10, 866-873 (2008).

19. Lancaster, J. R., Jr. \& Xie, K. Tumors face NO problems? Cancer Res 66, 6459-6462 (2006).

20. Maejima, Y., Adachi, S., Morikawa, K., Ito, H. \& Isobe, M. Nitric oxide inhibits myocardial apoptosis by preventing caspase-3 activity via S-nitrosylation. Journal of molecular and cellular cardiology 38, 163-174 (2005).

21. Juang, S. H. et al. Suppression of tumorigenicity and metastasis of human renal carcinoma cells by infection with retroviral vectors harboring the murine inducible nitric oxide synthase gene. Human gene therapy 9, 845-854 (1998)
22. Juang, S. H. et al. Use of retroviral vectors encoding murine inducible nitric oxide synthase gene to suppress tumorigenicity and cancer metastasis of murine melanoma. Cancer biotherapy \& radiopharmaceuticals 12, 167-175 (1997)

23. Xie, K. et al. Transfection with the inducible nitric oxide synthase gene suppresses tumorigenicity and abrogates metastasis by K-1735 murine melanoma cells. J Exp Med 181, 1333-1343 (1995).

24. Windbichler, G. H. et al. Interferon-gamma in the first-line therapy of ovarian cancer: a randomized phase III trial. British journal of cancer 82, 1138-1144 (2000).

25. Sebolt-Leopold, J. S. \& Herrera, R. Targeting the mitogen-activated protein kinase cascade to treat cancer. Nature reviews. Cancer 4, 937-947 (2004).

26. Olivier, M., Bautista, S., Valles, H. \& Theillet, C. Relaxed cell-cycle arrests and propagation of unrepaired chromosomal damage in cancer cell lines with wildtype p53. Mol Carcinogen 23, 1-12 (1998).

27. Wendt, J. et al. Induction of p21CIP/WAF-1 and G2 arrest by ionizing irradiation impedes caspase-3-mediated apoptosis in human carcinoma cells. Oncogene 25, 972-980 (2006)

28. Fujiuchi, N. et al. Requirement of IFI16 for the maximal activation of p53 induced by ionizing radiation. The Journal of biological chemistry 279, 20339-20344 (2004).

29. Wang, T. et al. The role of peroxiredoxin II in radiation-resistant MCF-7 breast cancer cells. Cancer research 65, 10338-10346 (2005).

30. Janicke, R. U. et al. Ionizing radiation but not anticancer drugs causes cell cycle arrest and failure to activate the mitochondrial death pathway in MCF-7 breast carcinoma cells. Oncogene 20, 5043-5053 (2001).

31. Treisman, R. Regulation of transcription by MAP kinase cascades. Current opinion in cell biology 8, 205-215 (1996).

32. Ahn, N. G. PORE-ing over ERK substrates. Nature structural \& molecular biology 16, 1004-1005 (2009)

33. Zehorai, E., Yao, Z., Plotnikov, A. \& Seger, R. The subcellular localization of MEK and ERK--a novel nuclear translocation signal (NTS) paves a way to the nucleus. Molecular and cellular endocrinology 314, 213-220 (2010).

\section{Acknowledgements}

The work was supported by the National Natural Science Foundation of China (Project $31070764,81273527,91013015$ and 81121062).

\section{Author contributions}

F.X.J. performed the experiments and contributed to the writing of the manuscript. S.T.Z. discussed the data and wrote the manuscript. B.Y.C. collaborated in the ERK-mutant experiment. D.S., Z.W., L.Y. and S.P.P. commented on the manuscript.

\section{Additional information}

Supplementary information accompanies this paper at http://www.nature.com/ scientificreports

Competing financial interests: The authors declare no competing financial interests.

License: This work is licensed under a Creative Commons

Attribution-NonCommercial-NoDerivs 3.0 Unported License. To view a copy of this license, visit http://creativecommons.org/licenses/by-nc-nd/3.0/

How to cite this article: FENG, X. et al. S-nitrosylation of ERK inhibits ERK phosphorylation and induces apoptosis. Sci. Rep. 3, 1814; DOI:10.1038/srep01814 (2013). 